\title{
Automutilaciones en pacientes psicóticos: reporte de dos casos inusuales.
}

\author{
Two rare cases of self-mutilation in psychotic patients. \\ Carla Cortez-Vergara1 ${ }^{1}$, Benilde C. Tirado-Hurtado², Patricia Núñez-Moscoso ${ }^{3}$, Lizardo Cruzado ${ }^{4}$
}

\section{RESUMEN}

Las automutilaciones que son objeto de atención psiquiátrica suelen ser atribuibles a enfermedades mentales graves y acarrean alteraciones anatómicas, funcionales y estéticas importantes: las más reportadas en la literatura son aquellas más severas (como las enucleaciones oculares y las autocastraciones), seguramente por lo llamativo de su presentación, pero existen otras automutilaciones que también pueden ser objeto de atención clínica. A partir de dos casos infrecuentes: automutilación de la mama en una mujer y de los pabellones auriculares de un varón, con diagnóstico de esquizofrenia ambos, revisamos la bibliografía pertinente y llamamos la atención sobre estos episodios, en los que la distorsión de la imagen y esquema corporal asomaron como factores relacionados al acto autolesivo, a diferencia de los factores de riesgo más comúnmente señalados en la literatura.

PALABRAS CLAVE: Esquizofrenia, automutilación, imagen corporal.

\section{SUMMARY}

Self-mutilations that require psychiatric care are often attributable to serious mental illnesses and produce important anatomic, functional and aesthetic alterations: the most reported in the literature are those of greater severity (such as eye enucleation and self-castration), probably because of their striking appearance. However, there are other selfmutilations that can also be subject of clinical care. From two rare cases of self-mutilation: the breast self-mutilation of a woman and the pinnae self-mutilation of a young men, both diagnosed with schizophrenia, we reviewed the relevant literature and call attention to these episodes, in which the image and body scheme distortion appeared as factors related to self-injurious acts, unlike the risk factors commonly found in the literature.

KEY WORDS: Schizophrenia, self-mutilation, body image.

1 Médica residente de Psiquiatría. Universidad Nacional Mayor de San Marcos. Instituto Nacional de Salud Mental Honorio Delgado - Hideyo Noguchi. Lima, Perú.

2 Médica residente de Psiquiatría. Universidad Nacional Mayor de San Marcos. Hospital Víctor Larco Herrera. Lima, Perú.

3 Médica psiquiatra. Departamento de Emergencia. Instituto Nacional de Salud Mental Honorio Delgado Hideyo Noguchi. Lima, Perú.

4 Médico psiquiatra. Facultad de Medicina Alberto Hurtado. Universidad Peruana Cayetano Heredia. Departamento de Emergencia. Instituto Nacional de Salud Mental Honorio Delgado - Hideyo Noguchi. Lima, Perú. 


\section{INTRODUCCIÓN}

No existe una clasificación consensuada de las lesiones autoinfligidas pero una de las tipologías más usadas las categoriza en: a) autolesiones estereotipadas (por ejemplo, las de pacientes oligofrénicos que se golpean repetidamente o se muerden a sí mismos), b) autolesiones compulsivas (como la tricotilomanía, onicofagia y dermatotilomanía), c) autolesiones impulsivas (cortes y laceraciones en la piel, frecuentes en algunos trastornos de personalidad) y d) automutilaciones, es decir, autolesiones de mayor envergadura (1). En ese tenor, la conducta automutilatoria conlleva destrucción tisular importante pero no posee propósito suicida y es socialmente inaceptable. Las variedades más frecuentes son la enucleación ocular, la castración, las amputaciones de extremidades y lesiones de otros órganos como nariz, lengua, boca, etc. Usualmente se ha adjudicado la mayoría de conductas automutilatorias a pacientes psicóticos (con diagnóstico de psicosis breve, esquizofrenia en estadio agudo, manía o melancolía) aunque también se han registrado automutilaciones en intoxicados por drogas, personalidades anormales, individuos con trastorno de la identidad sexual y otros casos circunscritos (2).

Apenas existen alrededor de un centenar de casos reportados de enucleación ocular y una cifra similar correspondiente a automutilaciones genitales a lo largo de más de un siglo. Aunque se han comunicado automutilaciones reiterativas parecen ser muy excepcionales: lo usual es un único acto automutilatorio en cada individuo afectado (3). Las lesiones mamarias, incluyendo la ablación de pezones, son muy infrecuentes (4). Asimismo, las mutilaciones de los pabellones auriculares se consideran entre las más raras (5).

Reportamos los casos de una mujer que se mutiló uno de sus pezones y un varón que se mutiló segmentos de ambas orejas, y hacemos un breve comentario de la literatura especializada.

\section{CASO CLÍNICO 1}

Mujer de 32 años sin antecedentes de importancia. Desde la adolescencia exhibió retraimiento social, lenguaje pobre y abandono de sus actividades. Solo tuvo una pareja sexual y no tenía problemas en su identidad de género. Hasta hace cinco años, fecha en que falleció su madre, la paciente no realizaba actividades productivas y se limitaba a acompañar a su progenitora actuando "como si fuera una niña pequeña". Hace tres años presentó fugas del hogar, soliloquios y risas inmotivadas; negaba además la muerte de la madre. Refería tener un "uñero" en la vulva que habría sido puesto en sus alimentos y de donde lo ingirió: deseaba corregir tal "defecto" cortándose los labios menores pese a las explicaciones de que sus genitales eran normales. También atribuía a dicho "uñero" su apetito incrementado y la necesidad de respirar por la boca pero no explicaba esta peregrina afirmación. Nunca recibió tratamiento psiquiátrico.

Acudió por emergencia tras cercenarse el pezón de la mama derecha con un cuchillo. Se sintió compelida porque el "uñero" la obligaba a respirar por la boca y "quería ver qué pasaba" al cortarse el pezón; el dolor que sintió no le pareció intolerable. Luego del acto arrojó el tejido residual y prorrumpió en risotadas: "me da risa la sangre", explicaba. En los días previos estaba inquieta, decía que un hombre entraba a espiar y robar su casa; negaba alucinaciones de cualquier tipo, incluyendo las auditivas imperativas.

Al examen se encontró una mujer obesa, pueril, presentaba delusiones pobremente sistematizadas de tipo somático y paranoide. Admitió alucinaciones verbales esporádicas que le decían: "loca". Su lenguaje era pobre, disgregado, con pararrespuestas. $\mathrm{Su}$ acto mutilatorio le producía hilaridad y carecía de conciencia de enfermedad. Tenía propósitos de cortarse el "uñero" en la vulva aunque la limitaba "el no tener puntería". En el examen físico no hubo anomalías aparte de la mutilación del pezón -que recibió sutura- ni en sus genitales externos.

En su historia clínica se registró el diagnóstico de esquizofrenia indiferenciada e inició tratamiento con $4 \mathrm{mg}$ diarios de risperidona, lográndose así la disminución de su sintomatología psicótica aunque sin desaparición completa del propósito de lacerarse sus genitales.

\section{CASO CLÍNICO 2}

Varón de 25 años de edad, desde su infancia fue tímido y lo hostilizaban llamándole "orejón". Hace ocho años, al terminar la educación secundaria, presentó conductas de aislamiento, irritabilidad, suspicacia, descuido de su ornato personal y mengua de rendimiento académico, llegando a abandonar toda actividad productiva y recluyéndose. Hace dos años se agravó: miccionaba en lugares inusitados, se embadurnaba con crema dental el bigote y la barba 
"para respirar mejor", además agredía a su hermana y la acusaba de haberle hecho brujería. Se hacía cortes superficiales en los brazos "para extraer a los fantasmitas de la muerte que le picaban el cuerpo" aunque a veces decía que lo hacía para "parecerse a Bruce Lee". Hace un año se evidenció asimismo que se había cortado -no se pudo estipular la fechaconsiderables segmentos de piel y cartílago en el borde superior de ambas orejas lo que justificaba alegando que ahora sus orejas sí eran simétricas y se alegraba pues ya no sería "orejón". En los últimos meses su condición empeoró: con mayor frecuencia se infería cortes en los antebrazos y se golpeaba la cabeza para no oír a "los fantasmitas", por lo que fue hospitalizado. Se le halló con varias cicatrices lineales y queloides en el antebrazo izquierdo y con los conductos auditivos taponados con algodón, además ocultaba bajo su larga melena sus orejas mutiladas. No tenía ánimo depresivo, ni ideas suicidas. Su sintomatología psicótica era prominente (delusiones de daño, enajenación del pensamiento, alucinaciones auditivas -que no eran imperativas- y también alucinaciones táctiles), y mantenía propósitos de lacerarse los antebrazos para "sacar los fantasmitas" que corrían por sus extremidades; carecía de conciencia de enfermedad. Aunque la remisión de la ideación autolesiva se logró al principio del tratamiento, el resto de su evolución fue muy lentamente favorable. Se le diagnosticó esquizofrenia paranoide y se mantiene estable desde hace 6 meses con $400 \mathrm{mg}$ diarios de clozapina (antes tuvo mala respuesta al haloperidol y a la risperidona), pero su tratamiento es irregular.

\section{DISCUSIÓN}

Aproximadamente el $11 \%$ de pacientes con esquizofrenia se autolesiona en algún momento entre el inicio de su psicosis y su primera atención de salud mental (6). Se estima una incidencia de un caso de automutilación mayor por cada cuatro millones de personas aunque hay un gran subregistro y solo una minoría de casos -usualmente los más graves- son los reportados en la literatura (3). Son factores de riesgo de automutilación las delusiones místicas y de culpa respecto a la sexualidad, y las alucinaciones imperativas, sobre todo en cuadros de esquizofrenia paranoide; también la historia de hospitalizaciones psiquiátricas previas, el consumo de sustancias psicoactivas y síntomas prevalentes de impulsividad y depresión $(1,2,6,7)$. Sumada a los cuadros psicóticos o de intoxicación por sustancias, también la disforia de género (transexualismo) explica varios casos de automutilación genital $(1,2)$. Otros factores predictores de la automutilación son un cambio drástico, voluntario y reciente en la apariencia del paciente y las autolesiones previas. Lamentablemente, en general las automutilaciones son difíciles de prevenir (1).

Honorio Delgado caracterizó a las automutilaciones como tributarias de una perturbación de las tendencias instintivas, específicamente de aquellas dirigidas a la defensa y posesión del cuerpo. Asimismo, resaltó el peculiar estado de indiferencia emocional en que se hallan los pacientes con esquizofrenia cuando se mutilan, como en los casos que aquí reportamos -a diferencia del ánimo colérico, propio de los epilépticos; o del ánimo desesperado y culposo de los melancólicos- (8). Sin embargo, la calma exhibida por muchos pacientes luego de la mutilación sugiere que tal conducta podría resolver o al menos apaciguar temporalmente conflictos internos subyacentes (1).

Un punto en común en nuestros pacientes fue el escaso dolor experimentado durante sus actos mutilatorios. En los trabajos de Kraepelin y Bleuler ya se había descrito este fenómeno y clínicamente se lo presume asociado -pues los pacientes con esquizofrenia no reportan el dolor esperable a su patología orgánica- a una mayor tasa de complicaciones médico-quirúrgicas en ellos; a la fecha, sin embargo, se carece de una explicación unitaria y total sobre la hipoalgesia esquizofrénica aunque ya se ha excluido al uso de antipsicóticos como explicación plausible de ella. Probablemente se trate de una respuesta sensorial aminorada, no solo ante el dolor sino ante diversas sensaciones corporales, y constituya un endofenotipo más que un síntoma asociado a la actividad de la psicosis, pues se ha encontrado incremento del umbral doloroso inclusive en personas sanas pero con historia familiar de esquizofrenia (9). La dopamina y el glutamato parecen ser los neurotransmisores involucrados en esta hipoalgesia pues participan en las vías neuronales del dolor y en aquellas relacionadas con la fisiopatología de la esquizofrenia (10). Específicamente, se postula una neurotrasmisión dopaminérgica incrementada en el estriado, lo que se correlacionaría con la agudización de la psicosis, que es cuando acontecen usualmente las automutilaciones. En cuanto a las automutilaciones en sí, la neurobiología involucra la patología psiquiátrica de base y es mucho más compleja e implica además disregulación serotoninérgica, gabaérgica y de opioides endógenos (11).

El primer reporte de mutilación mamaria en mujeres (4) fue el de una mujer con diagnósticos de 
esquizofrenia y alcoholismo, posteriormente suicida, aunque su mutilación aconteció mientras la psicosis se hallaba inactiva. Los autores resaltaron el conflicto con su femineidad en la génesis del acto y el correlato disociativo en que se produjo. Posteriormente se reportó (12) el caso de una mujer que se produjo laceraciones en pezones y genitales pues así "protegía" a su familia de sus perseguidores delusionales. El nuestro es el primer caso aislado de mutilación mamaria en una mujer con síntomas activos de psicosis.

La delusión dismorfofóbica respecto a los genitales externos ha sido reseñada en otros casos (12) aunque nuestra paciente no llegó a perpetrar dicha mutilación. Resalta en su caso la ausencia de la mayoría de factores de riesgo de mutilación descritos previamente. Asimismo destaca la peculiar desorganización del pensamiento y distorsión del esquema corporal que llevaba a nuestra paciente a ubicar un uñero en sus genitales y a conectar la vía digestiva con la respiratoria e inclusive con la glándula mamaria. Las alteraciones de la imagen corporal se han descrito repetidamente en personas con esquizofrenia pero no se ha profundizado en su posible relación con actos automutilatorios (13, 14).

Aunque las alucinaciones auditivas se cuentan entre los síntomas más frecuentes de la esquizofrenia, sorprende que no existan pacientes con dicha patología que se inflijan lesiones en los oídos u orejas con el fallido propósito de atenuar el tormento de sus pseudopercepciones. La automutilación de la oreja izquierda del pintor Vincent Van Gogh (18531890), tal vez la más famosa de la historia en su tipo, con seguridad no correspondió a un diagnóstico de esquizofrenia. Los pocos casos reportados de automutilaciones de las orejas corresponden a personas con esquizofrenia y también a individuos con personalidad antisocial o limítrofe en condición de encarcelados y algunos de ellos tienen en común con nuestro paciente la preocupación dismorfofóbica respecto a sus pabellones auriculares, a los que juzgaban desproporcionadamente prominentes (15). Aquí nuevamente asoma el tema de la inconformidad con la imagen corporal como motivación para la mutilación, y que en el caso de nuestro paciente existía ya antes de la eclosión de la psicosis. Se ha registrado la influencia de la dismorfofobia, incluso de tipo no delusional, en conductas automutilatorias (16). También llama la atención que el paciente se produjese otras autolesiones (cortes en los antebrazos), a consecuencia de sus fenómenos alucinatorios, pues ello no es frecuente.
En el manejo del paciente con automutilaciones se ha señalado que existe algún riesgo de que el paciente intente "completar" su acto si éste fue parcial o totalmente frustro, por lo que deben extremarse las medidas para el control de su psicosis y agitación, incluyendo la sedación y contención mecánica (2). La valoración psiquiátrica debe ser presta y a la vez meticulosa si existe la posibilidad de reimplantar el órgano cercenado (pene, dedos, etc.) y en general suele ser aconsejable proceder con la cirugía pero con las precauciones del caso (1). Se ha señalado a la clozapina como antipsicótico con cierta efectividad diferenciada en el alivio de la conducta automutilatoria en personas con esquizofrenia (2). Otro aspecto imprescindible en el manejo de estos pacientes es atender la intensa contratransferencia hostil que pueden experimentar los cuidadores primarios y personal asistencial hacia el paciente que se ha automutilado $(17,18)$.

En conclusión, aunque los fenómenos de automutilación son raros, dadas sus ominosas consecuencias es necesario no descuidar su posible prevención, más aún cuando el acto mutilatorio es a su vez un fuerte predictor de suicidio posterior en pacientes con esquizofrenia. Se impone además profundizar en el estudio de la distorsión de la imagen y esquema corporal como factor de riesgo para conductas automutilatorias.

\section{Correspondencia:}

\section{Lizardo Cruzado}

Departamento de Emergencia. Instituto Nacional de Salud Mental Honorio Delgado - Hideyo Noguchi.

Av. Eloy Espinoza 709. Urb. Palao.

San Martín de Porres. Lima, Perú.

Correo electrónico: lizardo_cruzado@yahoo.com

\section{Declaración de conflictos de interés:}

Ninguno por declarar.

\section{REFERENCIAS BIBLIOGRÁFICAS}

1. Favazza AR. The coming of age of self-mutilation. J Nerv Ment Dis. 1998; 186: 259-268.

2. Rao KN, Sudarshan CY, Begum S. Selfinjurious behavior: A clinical appraisal. Indian J Psychiatry. 2008; 50: 288-297.

3. Large M, Babidge N, Andrews D, Storey P, Nielssen O. Major self-mutilation in the first episode of psychosis. Schizophr Bull. 2009; 35: 1012-1021.

4. Coons PM, Ascher-Svanum H, Bellis K. Selfamputation of the female breast. Psychosomatics. 1986; 27: 667-668. 
5. Silva JA, Leong GB, Weinstock RA. A case of skin and ear self-mutilation. Psychosomatics. 1989; 30: 228-230.

6. Harvey SB, Dean K, Morgan C, Walsh E, Demjaha A, Dazzan P, et al. Self-harm in first-episode psychosis. Br J Psychiatry. 2008; 192: 178-184.

7. Pluck G, Lekka NP, Sarkar S, Lee KH, Bath PA, Sharif O, Woodruff PDR. Clinical and neuropsycholgical aspects of non-fatal self-harm in schizophrenia. European Psychiatry. 2012, http:// dx.doi.org/10.1016/j.eurpsy.2012.08.003

8. Delgado H. Contribuciones a la Psicología y la Psicopatología. Lima: Ediciones Peri-Psyche; 1962.

9. Potvin S, Marchand S. Hypoalgesia in schizophrenia is independent of antipsychotic drugs: A systematic quantitative review of experimental studies. Pain. 2008; 138: 70-78.

10. Singh MK, Giles LL, Nasrallah HA. Pain insensitivity in schizophrenia: Trait or state marker? J Psychiatr Pract. 2006; 12: 90-102.

11. Winchel RM, Stanley M. Self-injurious behavior: a review of the behavior and biology of self-mutilation. Am J Psychiatry. 1991; 148: 306-17.
12. Vohra AK. Genital and nipple self-mutilation in a female schizophrenic patient. German J Psychiatry. 2010; 13: 91-93.

13. Priebe S, Röhricht R. Specific body image pathology in acute schizophrenia. Psychiatry Res. 2001; 101: 289-301.

14. Millán-González R. Mutilación genital en paciente transexual: ¿manifestación de un espectro patológico imprecisamente definido? Rev Colomb Psiquiat. 2010; 39: 624-634.

15. Alroe CJ, Gunda V. Self-amputation of the ear: three men amputate four ears within five months. Aust NZ J Psychiatry. 1995; 29: 508-512.

16. Conejo-García A, Moreno-Pinilla M, Crespo-Hervás D, Saiz-Ruiz J. Complicaciones de la dismorfofobia. Descripción de un caso de automutilación. Actas Esp Psiquiatr. 2006; 34: 202-205.

17. Simopoulos EF, Trinidad AC. Two cases of male genital self-mutilation: an examination of liaison dynamics. Psychosomatics. 2012; 53: 178-180.

18. Munerato MC, Pozatti-Moure S, Machado V, GriloGomes F. Self-mutilation of tongue and lip in a patient with simple schizophrenia. Clin Med Res. 2011; 9: 42-45. 